Rev. Inst. Flor. v. 30 n. 1 p. 87-94 jun. 2018

http://dx.doi.org/10.24278/2178-5031.201830107

ISSN impresso 0103-2674/on-line 2178-5031

\title{
MORFOLOGIA E EMERGÊNCIA DE PLÂNTULAS DE Oenocarpus bataua MARTIUS EM DIFERENTES SUBSTRATOS E LUMINOSIDADES ${ }^{1}$
}

\section{MORPHOLOGY AND EMERGENCY GERMINATION OF Oenocarpus bataua MARTIUS SEEDLINGS IN DIFFERENT SUBSTRATES AND LUMINOSITIES}

\author{
Auriane da Conceição Dutra da SILVA, ${ }^{2,4}$ Ataiza de Andrade SOUSA²; Cassia Rejane do NASCIMENTO²; \\ Oscar Jose SMIDERLE ${ }^{3}$
}

\begin{abstract}
RESUMO - Objetivou-se neste trabalho descrever morfologicamente o pirênio e caracterizar os estágios morfológicos da plântula de Oenocarpus bataua durante a germinação e emergência e verificar o efeito do substrato e ambiente na emergência de pirênios de Oenocarpus bataua. Foram utilizados frutos obtidos de mistura de matrizes. Os frutos maduros foram submetidos à extração do epicarpo e mesocarpo. No primeiro ensaio, foi realizada biometria em 200 pirênios e semeados em vermiculita para caracterização morfológica das plântulas. No segundo ensaio, foi testada a influência de diferentes substratos (vermiculita, Plantmax ${ }^{\circledR}$, solo natural e areia) e condições de luz (a pleno sol e em ambiente de viveiro), sobre a emergência das plântulas. As variáveis analisadas foram: porcentagem de emergência e tempo médio de emergência. O pirênio é ovalado, o endocarpo é formado por fibras com coloração amarelo-clara e marrom, estando aderidas ao tegumento, possui único poro germinativo. A germinação é adjacente ligulada e hipógea. A completa expansão da primeira folha bífida dá-se aos 95 dias. Os substratos mais adequados para a emergência de plântulas de Oenocarpus bataua são vermiculita, areia e Plantmax ${ }^{\circledR}$.
\end{abstract}

Palavras-chave: Patauá; Pleno sol; Sombreamento.

\begin{abstract}
This work aimed to describe morphologically the pyrenes and characterize morphological stages of the Oenocarpus bataua seedling during germination and emergence, and to verify the substrate and environment effects in the emergence of Oenocarpus bataua Seeds were used obtained from mixing matrix. The ripe fruit were submitted to the extraction of epicarp and mesocarp. In the first experiment was conducted biometrics in 200 seeds and sown in vermiculite for morphological characterization of the seedlings. In the second experiment the influence of different substrates (vermiculite, Plantmax ${ }^{\circledR}$, natural soil and sand) and light conditions (full sun and nursery environment) on the emergence of seedlings were tested. The analyzed variables were: percentage of emergency and mean time of emergency. The pyrene is oval, the endocarp is formed by fibers with light yellow and brown color being adhered to the integument, it has only germinative pore. The germination is adjacent ligated and hypogeous. The complete expansion of the first bifid leaf occurs at 95 days. The most suitable substrates for emergence of Oenocarpus bataua seedlings are vermiculite, sand and Plantmax ${ }^{\circledR}$.
\end{abstract}

Keywords: Patawa; Full Sun; Shading.

\footnotetext{
${ }^{1}$ Recebido para análise em 07.08.2017. Aceito para publicação em 10.06.2018.

${ }^{2}$ Programa de Pós-Graduação em Agronomia, Universidade Federal de Roraima (POSAGRO/UFRR), Monte Cristo, CEP: 69300-000, Boa Vista-RR, Brasil.

${ }^{3}$ Embrapa Roraima, Rodovia BR 174, Km 8, Distrito Industrial, Caixa Postal: 133, CEP: 69301-970, Boa Vista, RR, Brasil.

${ }^{4}$ Autor para correspondência: Auriane da Conceição Dutra da Silva - Silvaauriane@hotmail.com
} 
SILVA, A.C.D. et al. Morfologia e emergência de plântulas de $O$. bataua em diferentes substratos e luminosidades.

\section{INTRODUÇÃO}

A palmeira Oenocarpus bataua Mart., também conhecida como patauá é uma frutífera não cultivada comercialmente, natural da floresta úmida de várzeas e galerias, tanto inundáveis como de terra firme de toda a região Amazônica, é uma das palmeiras mais utilizadas na região. O principal recurso obtido da espécie são os frutos colhidos de populações silvestres para consumo humano (Isaza et al., 2016). Devido ao alto conteúdo de ácido oleico, comparável ao do azeite de oliva, os frutos de patauá são uma ótima fonte de óleo monoinsaturado (Hidalgo et al., 2016).

A propagação da maioria das palmeiras é de forma sexuada, no entanto, de maneira geral, a germinação é lenta, desuniforme e influenciada por vários fatores, como estágio de maturação, presença ou não de pericarpo, tempo entre colheita e semeadura, dormência física, temperatura do ambiente e substrato, entre outros (Pivetta et al., 2008). O desenvolvimento das estruturas básicas do processo germinativo é bastante peculiar na família Arecaceae, podendo diferir entre espécies, desta forma, o conhecimento dos estágios morfológicos durante a germinação das sementes de cada espécie de palmeira é imprescindível para auxiliar na análise do ciclo vegetativo, podendo fornecer subsídios à interpretação de testes de germinação e também auxiliar os estudos de taxonomia e ecologia (Taveira et al., 2013; Pereira et al., 2014).

Dentro da tecnologia e análise de sementes, o teste de germinação é o suporte para todas as outras análises e experimentos, e o conhecimento das plântulas e de suas estruturas (Da Luz et al., 2012). Dentre os fatores que afetam a germinação, destaca-se o substrato, a escolha deve ser feita em função da espécie e considerando algumas das características da semente, tais como anatomia, necessidade de água e luz e, ainda, facilidade da contagem e avaliação das plântulas, conforme consta em Brasil (2009).

A vermiculita vem sendo recomendada como substrato ideal para germinação de sementes de Arecaceae, que em geral tem grandes dimensões e formato arredondado, permitindo o desenvolvimento mais adequado de plântulas durante o teste de germinação, em função do maior contato estabelecido entre a semente e o substrato (Figliolia et al., 1993). No entanto diversos materiais orgânicos e inorgânicos têm sido utilizados na formulação de substratos para a produção de mudas, havendo necessidade de determinar os mais apropriados para cada espécie (Alves et al., 2008)

Durante o processo de germinação, a temperatura afeta a velocidade de absorção de água pelas sementes e pode alterar, entre outros aspectos, a porcentagem, velocidade e a uniformidade de germinação. Há uma amplitude de temperatura para que ocorra a germinação, a qual varia com cada espécie, existindo temperatura máxima e mínima, acima e abaixo das quais a germinação não ocorre. A temperatura ideal para a germinação geralmente está relacionada à faixa de temperatura típica do local de ocorrência da espécie (Rodrigues et al., 2014).

Conforme Oliveira et al. (2009), o ambiente de cultivo ou produção de mudas pode afetar positiva ou negativamente a qualidade das plantas. Estes autores salientaram que o sombreamento, proporcionado pelo emprego de casas de vegetação, pode ser utilizado para auxiliar no controle excessivo de temperatura, destacando que a redução da radiação solar, com telas, pode diminuir a temperatura do ambiente em até cinco graus Celsius, entre outros benefícios.

Objetivou-se com esse trabalho descrever morfologicamente o pirênio (endocarpo aderido à semente), caracterizar os estágios morfológicos da plântula durante a germinação e verificar o efeito de substratos e ambientes na emergência de plântulas de Oenocarpus bataua.

\section{MATERIAL E MÉTODOS}

O presente trabalho foi desenvolvido no Laboratório de Sementes e no Viveiro de Plantas Nativas do Centro de Ciências Agrárias da Universidade Federal de Roraima (UFRR). Os pirênios foram adquiridos em uma pequena fábrica de polpas em Boa Vista-RR, após a extração do epicarpo e do mesocarpo em despolpadora mecânica. Os diásporos foram selecionados manualmente, descartando aqueles mal formados ou parcialmente danificados. Posteriormente foram realizadas: limpeza, desinfestação dos diásporos em hipoclorito de sódio a $6 \%$ por 10 minutos e, em seguida, lavagem com água destilada. 
SILVA, A.C.D. et al. Morfologia e emergência de plântulas de $O$. bataua em diferentes substratos e luminosidades.

\section{Ensaio 1: Morfometria e Morfologia}

Para caracterização morfométrica, foi utilizada amostra de 200 pirênios, analisados conforme: forma, coloração, tamanho (diâmetro longitudinal e equatorial) e o peso da matéria seca. Os resultados foram expressos em porcentagem, com base no peso da amostra úmida). O embrião foi avaliado quanto à forma, tamanho, coloração e posição.

Para obter o peso da matéria seca, quatro repetições com 10 pirênios cada foram colocadas em estufa a $105 \pm 3{ }^{\circ} \mathrm{C}$ por 24 horas de acordo com as Regras para Análise de Sementes (BRASIL, 2009). A umidade foi determinada em base úmida e o grau de umidade foi obtido pela equação: $\left(\left(\mathrm{P}_{1}-\mathrm{P}_{2}\right) / \mathrm{P}_{1}\right) * 100=\mathrm{UR}$, onde $\mathrm{P}_{1}$ : massa da matéria fresca; $\mathrm{P}_{2}$ : massa da matéria seca; UR: umidade relativa em $\%$.

Para descrição morfológica da plântula, foram semeados 200 pirênios em bandejas de plástico com substrato vermiculita, a $2 \mathrm{~cm}$ de profundidade, sob sombrite $50 \%$ e irrigação por nebulização intermitente. O acompanhamento do processo germinativo e do desenvolvimento da plântula foi realizado a cada dois dias, desde a emergência do pecíolo cotiledonar até a completa expansão do primeiro eofilo (primeira folha bífida).

\section{Ensaio 2: Efeito do substrato e luminosidade}

Foram utilizados quatro substratos (vermiculita, composto orgânico comercial $\left(\right.$ Plantmax $\left.^{\circledR}\right)$, solo natural (classificado como Latossolo Vermelho-Amarelo, de baixa fertilidade natural $02^{\circ} 56^{\prime} 43,5^{\prime \prime} \mathrm{N} 60^{\circ} 43^{\prime} 00,8^{\prime \prime} \mathrm{W}$, coletado na camada entre 0 e $15 \mathrm{~cm}$ de profundidade)) e dois ambientes (pleno sol e viveiro telado com sombrite a $50 \%$ de luminosidade), com cinco repetições de 20 pirênios.

Os pirênios foram semeados a $2 \mathrm{~cm}$ de profundidade em bandejas plásticas $(28 \times 40 \times 15 \mathrm{~cm})$ perfuradas no fundo e preenchidas com $2 / 3$ da capacidade de seu volume, com os respectivos substratos. Estes foram umedecidos com quantidade de água equivalente a $60 \%$ da capacidade de retenção (BRASIL, 2009). A reposição hídrica foi feita diariamente, mantendo-a próximo ao nível inicial.

A contagem da emergência foi iniciada 34 dias após a semeadura (DAS), sendo realizada todo dia, até a estabilização do processo (50 DAS). Os dados obtidos foram utilizados para o cálculo das porcentagens de emergência (\%) e tempo médio de emergência, segundo as metodologias descritas nas Regras para Análise de Sementes (BRASIL, 2009).

Os dados obtidos foram submetidos à análise de variância pelo teste $\mathrm{F}(\mathrm{p} \leq 0,05)$; quando significativo, foram realizados testes de médias dos tratamentos, utilizando o teste de Tukey para comparação entre substratos e o teste $\mathrm{T}$ para comparação entre regimes de luz, ambos a $5 \%$ de probabilidade $(\mathrm{p} \leq 0,05)$.

\section{RESULTADOS E DISCUSSÃO}

\section{Ensaio1}

O pirênio de Oenocarpus bataua é ovalado, pesa em média 4,32 $\mathrm{g}$ de massa fresca e $2,86 \mathrm{~g}$ de massa seca, correspondendo a 56,26\% de umidade, dados apresentados na Tabela 1.

Durante o processo de maturação, as sementes passam por modificações físicas (tamanho, coloração e teor de água), bioquímicas (açúcares, proteínas, óleo, ácidos graxos) e fisiológicas (germinação, vigor, massa seca). Essas modificações são influenciadas por fatores genéticos e ambientais até atingir o ponto ideal de colheita, quando apresentam a capacidade máxima de germinação e vigor (Martins et al., 2008). O teor de água inicial de sementes fisiologicamente maduras em parte, pode ser atribuído ao material genético, bem como aos dias decorridos após a colheita dos frutos até o início do experimento (Martins et al., 2009).

Tabela 1. Dados morfométricos obtidos de 200 pirênios de Oenocarpus bataua Mart.

Table 1. Morphometric data obtained from 200 pyrenes of Oenocarpus bataua Mart.

\begin{tabular}{lccccc}
\hline \multicolumn{1}{c}{ Pirênio $(\mathrm{cm})$} & Amplitude & Média & Mediana & Desvio Padrão & CV (\%) \\
\hline Diâmetro longitudinal & $2,55-3,00$ & 2,69 & 2,71 & 0,12 & 4,46 \\
Diâmetro equatorial maior & $1,40-1,75$ & 1,57 & 1,58 & 0,07 & 4,45 \\
Diâmetro equatorial menor & $1,35-1,70$ & 1,53 & 1,54 & 0,07 & 4,57 \\
Massa fresca & $3,7-5,1$ & 4,32 & 4,30 & 0,08 & 4,45 \\
Massa seca & $1,9-3,5$ & 2,86 & 2,91 & 0,09 & 4,54 \\
\hline
\end{tabular}


SILVA, A.C.D. et al. Morfologia e emergência de plântulas de $O$. bataua em diferentes substratos e luminosidades.

O endocarpo é formado por fibras com coloração amarelo-clara e marrom, estando aderidas ao tegumento. Este é uma fina camada de coloração marrom-alaranjada protegendo um endosperma sólido formado por um tecido denso, enrijecido e homogêneo. Essas características são típicas das Arecaceae. Oenocarpus minor Mart., por exemplo, é formado por vários extratos de fibras que, observados a olho nu, mostram duas colorações, amarelo-clara e marrom, estando parcialmente aderidas ao tegumento (Mendonça et al., 2008). Segundo Carvalho e Aoyama (2007), em pirênios de Caryota mitis Lour, o endocarpo de superfície lisa tem coloração marrom.

O pirênio possui único poro germinativo, que corresponde à micrópila, sendo protegido por um tufo de fibras do endocarpo. O embrião é basal, indiviso, carnoso, de coloração amarelo pálida (Figura 1A), localizado abaixo do tegumento, consiste de um único cotilédone que envolve o eixo embrionário. Como em Oenocarpus bacaba Mart., Queiroz e Bianco (2009) descrevem essa espécie com único poro germinativo protegida pelo opérculo que é uma película que cobre o poro germinativo. Análogo a Oenocarpus bataua e Dypsis decaryi (Jum.), o embrião encontra-se localizado na parte central do pirênio e apresenta coloração creme (Bao et al., 2010).

A germinação de Oenocarpus bataua é adjacente ligulada, o cotilédone que não se eleva acima do nível do substrato durante o alongamento do epicótilo, ocorrendo o desenvolvimento da plântula adjacente ao pirênio. Aos 11 DAS, verificou-se o intumescimento do pecíolo cotiledonar, formando botão germinativo de aspecto cilíndrico e coloração esbranquiçada (Figura 1B).

O início da germinação de Oenocarpus bataua ocorreu a partir de nove dias após a semeadura (DAS) em virtude da pressão exercida pelo botão germinativo em crescimento. Em trabalhos realizados com sementes de Archontophoenix cunninghamii, destaca-se o início da germinação após 10 dias da instalação do experimento, em que o desenvolvimento tem seu início a partir de uma massa de células indiferenciadas na depressão micropilar (Charlo et al., 2006; Pivetta et al., 2008).
A raiz primária surgiu por volta de 13 DAS (Figura 1C), iniciando, a partir da região em que posteriormente ocorreu o desenvolvimento da lígula cotiledonar (Figura 1D), uma estrutura tubular, que se tornou evidente após o desenvolvimento da primeira bainha plumular. Aos 19 DAS, surgiu a primeira raiz adventícia localizada sob a primária (Figura 1E). Nessa fase é reduzida a presença de pelos absorventes (Figura 1G). Em seguida, aos 24 DAS, ocorreu o rompimento da lígula na região superior com coloração esverdeada, que corresponde à emissão da primeira bainha (Figura 1F). Na sequência, houve a emissão do ápice do eofilo aos 44 DAS (Figura 1J).

O surgimento da primeira raiz adventícia localizada sob a primária em Oenocarpus bataua é descrito para outras espécies de Arecaceae. Queiroz e Bianco (2009), estudando a germinação de Oenocarpus bacaba Mart., relataram a existência da raiz primária, geralmente transitória, sendo substituída pela primeira raiz adventícia.

A segunda bainha protetora surge a partir da primeira, que ocorreu aos $61 \mathrm{DAS}$, constituindo assim o primórdio caulinar (Figura $1 \mathrm{H}$ ), permitindo a emergência da folha primária. A primeira bainha é localizada próxima ao eixo embrionário e apresenta pequena extensão quando comparada com a segunda.

A plúmula é composta por duas folhas incompletas denominadas bainhas, que revestem a primeira folha juvenil completa, denominada de eofilo; o surgimento desta define o fim da fase plantular. A completa expansão da primeira folha bífida em plântulas de Oenocarpus pataua ocorreu aos 95 DAS, como também em Oenocarpus bacaba, em que a expansão completa do primeiro eofilo em plântulas deu-se aos 95 dias após a semeadura (Queiroz e Bianco, 2009).

Desde o início do processo germinativo até a formação da plântula normal, o diásporo está firmemente ligado à plântula por meio do pecíolo cotiledonar. A mesma estrutura foi observada por Silva et al. (2006a) em Bactris gasipaes e Charlo et al. (2006) em Archontophoenix alexandrae. A completa expansão da primeira folha bífida em plântulas de Oenocarpus bataua ocorre aos 95 DAS apresentando limbo de coloração verde-escura (Figura 1I). 

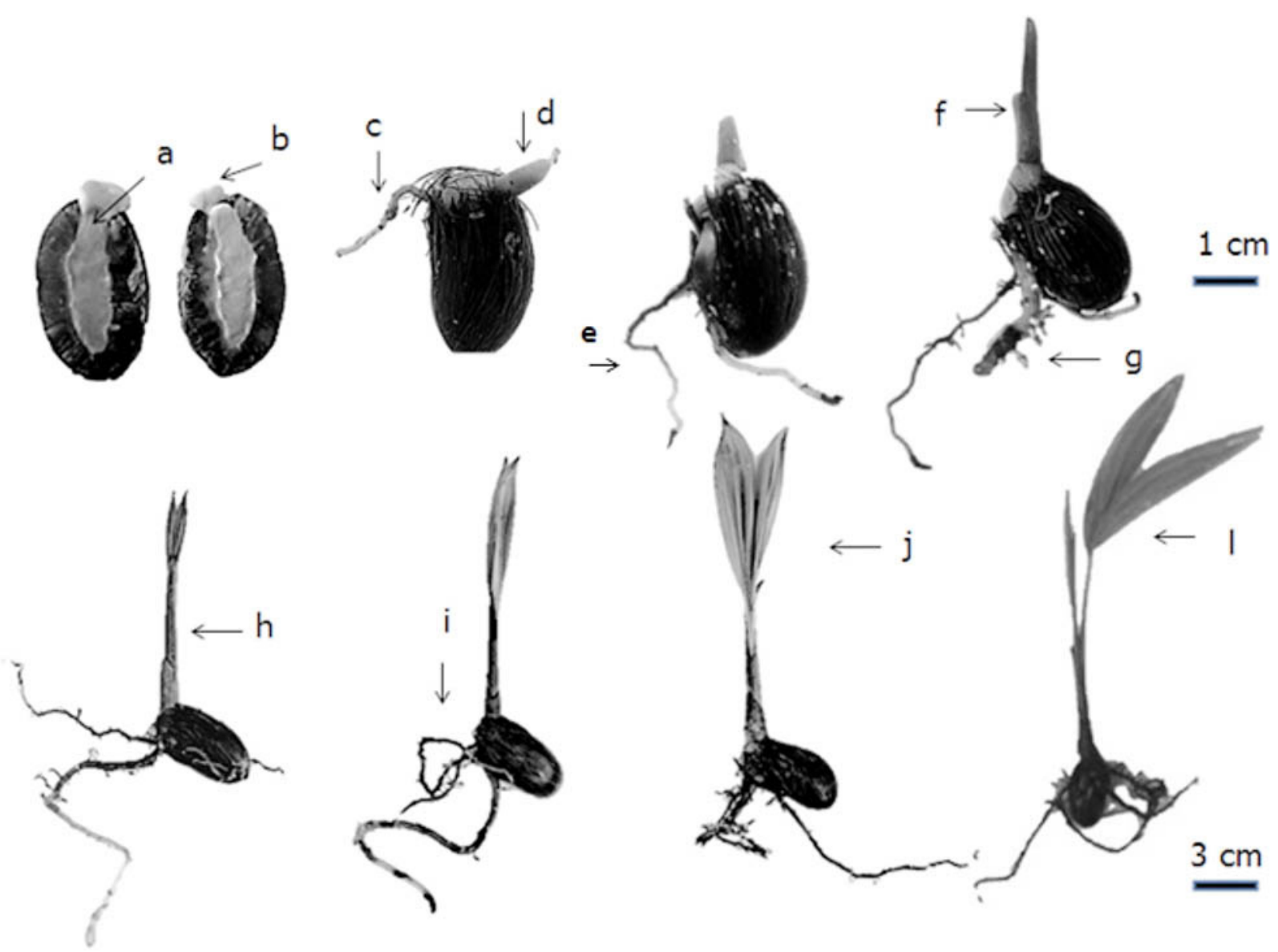

Figura 1. Fases iniciais da germinação, emergência e desenvolvimento de plântulas de Oenocarpus bataua Mart. Legenda: a - embrião; b - botão germinativo; c - raiz primária; d - lígula; e - raiz secundária; f - primeira bainha plumular; $g$ - pelos radiculares; $\mathrm{h}$ - segunda bainha plumular; $\mathrm{i}$ - raízes adventícias; $\mathrm{j}$ - primeiro eofilo; 1 - expansão completa do primeiro eofilo.

Figure 1. Initial stages of germination, emergence and development of Oenocarpus bataua Mart. Legend: a - embryo; b - germinative button; c - primary root; $\mathrm{d}$ - ligula; and - secondary root; f - first plumular sheath; $\mathrm{g}$ - root hairs; $\mathrm{h}$ - second plumular sheath; i - adventitious roots; $\mathrm{j}$ - first eofilo; 1 - full expansion of the first eofilo.

\section{Ensaio 2}

Em análises realizadas, observou-se que houve diferença significativa ao nível de $5 \%$ entre os substratos nas variáveis estudadas em pirênios de Oenocarpus bataua. Constatou-se que, em viveiro, o percentual de emergência de plântulas foi superior quando utilizado o substrato vermiculita, não diferindo significativamente do Plantmax ${ }^{\circledR}$ (Tabela 2). A pleno sol, observa-se número maior de emergência quando utilizadas vermiculita e areia. O substrato solo natural apresentou a menor porcentagem de germinação tanto no viveiro como a pleno sol $(43,40 \%$ e $46,20 \%$, respectivamente).
Em geral, altos índices de emergência são registrados em espécies de Arecaceae, similares aos resultados obtidos neste trabalho, entre elas: Oenocarpus minor Mart. com 95\%(Queiroze Bianco, 2009)e Bactris marajá com 90\% (Rodrigues et al., 2014) e Silva et al. (2007) também registraram resultados semelhantes com relação à germinação em Oenocarpus minor Mart., todos contendo areia como substrato e mantidos em viveiro telado.

Conforme as características dos substratos estudados, observaram-se diferentes respostas em função dos materiais utilizados. Neste sentido, de acordo com as características físicas, os substratos possuem diversos níveis de disponibilidade de água e estas características podem ter influenciado a velocidade de embebição e consequentemente a germinação (Silva et al., 2006a). 
SILVA, A.C.D. et al. Morfologia e emergência de plântulas de $O$. bataua em diferentes substratos e luminosidades.

Tabela 2. Porcentagem de emergência de plântulas (PE) e tempo médio de emergência (TME) de Oenocarpus bataua Mart. em diferentes substratos e ambientes com diferentes níveis de luminosidades (viveiro 50\% de luminosidade e pleno sol).

Table 2. Percentage of emergence of seedlings (PE) and mean time of emergence (TME) of Oenocarpus bataua Mart. in different substrates and environments with different levels of luminosity (nursery $50 \%$ of luminosity and full sun).

\begin{tabular}{|c|c|c|c|c|c|c|}
\hline Substrato & $\begin{array}{l}\text { Viveiro } \\
\text { PE (\%) }\end{array}$ & $\begin{array}{c}\text { Pleno sol } \\
\text { PE }(\%)\end{array}$ & $\mathrm{F}(\mathrm{P})$ & $\begin{array}{c}\text { Viveiro } \\
\text { TME (dia) }\end{array}$ & $\begin{array}{c}\text { Pleno sol } \\
\text { TME (dia) }\end{array}$ & $\mathrm{F}(\mathrm{P})$ \\
\hline Plantmax $^{\circledR}$ & $95,0 \mathrm{a}$ & $70,0 \mathrm{~b}$ & 0,042 & $41,48 \mathrm{a}$ & $44,86 \mathrm{~b}$ & 0,054 \\
\hline Areia & $59,0 \mathrm{~b}$ & $98,0 \mathrm{a}$ & 0,032 & $45,84 \mathrm{~b}$ & $44,04 \mathrm{~b}$ & 0,054 \\
\hline Vermiculita & $97,8 \mathrm{a}$ & $99,0 \mathrm{a}$ & 0,058 & $41,20 \mathrm{a}$ & $40,20 \mathrm{a}$ & 0,056 \\
\hline solo natural & $43,4 \mathrm{~b}$ & $46,2 \mathrm{c}$ & 0,056 & $49,40 \mathrm{c}$ & $48,20 \mathrm{c}$ & 0,064 \\
\hline
\end{tabular}

Médias seguidas das mesmas letras (na coluna) não diferem entre si ao nível de significância a 5\% de probabilidade pelo teste de Tukey.

Means followed by the same letters (in the column) do not differ among them at the level of significance at $5 \%$ of probability by the Tukey test.

De acordo com os resultados, verificou-se no substrato solo natural a menor porcentagem de germinação (43,4\% em pleno sol e 46,2\% em viveiro), provavelmente devido à baixa aeração do substrato. A maior proporção de partículas grossas no substrato em relação a partículas finas favorece maior espaço de aeração, enquanto que a menor proporção favorece a retenção de água, acarretando falta de oxigenação aos pirênios (Fermino, 2003).

Diversos autores realizaram pesquisas que permitem amplas informações, como em Archontophoenix cunninghamii, em que a vermiculita proporcionou maior porcentagem de emergência (81\%) comparada à areia e ao esfagno (Pivetta et al., 2008). Por outro lado, Jose et al. (2012) indicaram a areia como melhor substrato para germinação de sementes de Oenocarpus bacaba. Para Luz et al. (2008), não houve diferença na porcentagem de germinação para sementes de Dypsis decaryi (Jum.) nos substratos areia e vermiculita.

Enquanto Charlo et al. (2006) observaram maior porcentagem de emergência de Archontophoenix alexandrae quando o substrato utilizado foi Plantmax ${ }^{\circledR}$ comparado à areia e ao solo natural. Para este experimento, observaram-se resultados análogos para os substratos vermiculita e Plantmax ${ }^{\circledR}$ em viveiro e vermiculita e areia a pleno sol, com taxa de porcentagem próximo de $90 \%$.

No que diz respeito aos ambientes, em pleno sol, observou-se menor tempo médio de emergência em relação ao viveiro nos substratos areia, vermiculita e solo natural, ainda que não diferindo estatisticamente. Os pirênios, submetidos a ambientes com maior intensidade luminosa, consequentemente a maiores níveis e amplitudes de temperatura, apresentaram melhores resultados de emergência, indicando que esta condição é adequada para germinação de Oenocarpus bataua. Igualmente em Euterpe oleracea, os resultados indicaram menor germinação em ambiente protegido comparado a pleno sol (Gama et al., 2010).

A influência da luminosidade sobre a germinação é variável nas espécies de Arecaceae, por exemplo, em: Oenocarpus minor Mart. (Silva et al., 2006b) e Archontophoenix cunninghamii (Pivetta et al., 2008), as quais não apresentaram interação significativa entre os substratos e temperaturas com relação à porcentagem de germinação destas espécies.

Germinação rápida e uniforme seguida por imediata emergência das plântulas são características desejáveis na produção de mudas, visto que o menor tempo de germinação diminui o tempo de suscetibilidade a patógenos (Tabela 2). No entanto para Silva et al. (2006b), em Oenocarpus minor Mart., a areia possibilitou menor tempo médio de germinação que a vermiculita.

Igualmente em Copernicia hospita Martius, o tempo médio de germinação foi menor em ambiente a pleno sol que em casa de vegetação (Oliveira et al., 2009) e, em Copernicia prunifera, os autores obtiveram emergência mais rápida a pleno sol quando comparada à do sombreamento (Reis et al., 2011). 
SILVA, A.C.D. et al. Morfologia e emergência de plântulas de $O$. bataua em diferentes substratos e luminosidades.

\section{CONCLUSÕES}

O pirênio de Oenocarpus pataua Mart. é ovalado, o endocarpo é formado por fibras com coloração amarelo-clara e marrom, estando aderidas ao tegumento, possui único poro germinativo.

A germinação é adjacente ligulada e hipógea. Aos 11 dias após a semeadura, verifica-se o intumescimento do pecíolo cotiledonar, a raiz primária surge aos 13, a fase plantular se completa aos 95 dias após a semeadura.

Os substratos mais adequados para a emergência de plântulas de Oenocarpus bataua a pleno sol são areia e vermiculita. Para viveiro são vermiculita e Plantimax ${ }^{\circledR}$.

\section{REFERÊNCIAS BIBLIOGRÁFICAS}

ALVES, E.U. et al. Substratos para testes de emergência de plântulas e vigor de sementes de Erythrina velutina Willd., Fabaceae. Semina: Ciências Agrárias, v. 29, n. 1, p. 69-82, 2008. http://dx.doi.org/10.5433/16790359.2008v29n1p69.

BAO, F.; LUZ, P.B.; PAIVA SOBRINHO, S.; NEVES, L.G. Morfologia do diásporo e da plântula de Dypsis decaryi (Jum.) Beentje \& J. Dransf. (Arecaceae). Revista Trópica - Ciências Agrárias e Biológicas, v.4, n.3, p.3, 2010.

BRASIL. Ministério da Agricultura e reforma Agrária. Regras para análise de sementes. Brasília-DF: Departamento Nacional de Produção Vegetal - SNA/ DNPV/CLAV, 2009. 399 p.

CARVALHO, C.P.; AOYAMA, E.M. Morfologia de frutos, sementes, plântulas e germinação de Caryota mitis Lour. (Arecaceae). Revista Biociências, v. 13, p. 148-155, 2007.

CHARLO, H.C.O. et al. Aspectos morfológicos, germinação e desenvolvimento inicial de plântulas de Archontophoenix alexandrae (F. Mueller) H. Wendl. e Drude (Arecaceae) em diferentes substratos. Revista Árvore, v. 30, n. 06, p. 933-940, 2006. http://dx.doi. org/10.1590/S0100-67622006000600008.
DA LUZ, P.B. et al. Caracterização morfológica do diásporo e da plântula de Archontophoenix cunninghamii (Arecaceae). Revista Comunicata Scientiae, v. 3, n. 4, p. 244-248, 2012.

FERMINO, M.H. Métodos de análise para caracterização de física de substratos. 2003. 89 f. Tese (Doutorado em Fitotecnia) - Universidade Federal do Rio Grande do Sul, Porto Alegre.

FIGLIOLIA, M.B.; OLIVEIRA, E.C.; PINARODRIGUES, F.C.M. Analise de sementes. In: AGUIAR, I.B.; PIÑA-RODRIGUES, F.C.M.; FIGLIOLIA, M.B. (Coords.) Sementes florestais tropicais. Brasília: Abrates, 1993, p. 137-174.

GAMA, J.S.N. et al. Temperaturas e substratos para germinação e vigor de sementes de Euterpe oleracea Mart. Revista Ciência Agronômica, v. 41, n. 04, p. 664-670, 2010. http://dx.doi.org/10.1590/S180666902010000400021.

HIDALGO,P.S.P.; NUNOMURA, R.C.S.; NUNOMURA, S.M. Plantas oleaginosas amazônicas: química e atividade antioxidante de patauá (Oenocarpus bataua Mart.). Revista Virtual de Química, v. 8, n. 1, p. 130-140, 2016.

ISAZA, C. et al. Demografia de Oenocarpus bataua e implicações para a colheita sustentável de seus frutos na Amazônia ocidental. Ecologia populacional, v. 58, n. 3, p. 463-476, 2016.

JOSE, A.C.; ERASMO, E.A.L.; COUTINHO, A.B. Germinação e tolerância à dessecação de sementes de bacaba (Oenocarpus bacaba Mart.). Revista Brasileira de Sementes, v. 34, n. 4, p. 651-657, 2012. http:// dx.doi.org/10.1590/S0101-31222012000400017.

LUZ, P.B. et al. Germinação de Dypsis decaryi Beentje \& J. Dransf. (Arecaceae). Revista Ciência e Agrotecnologia, v. 32, n. 05, p. 1461-1466, 2008. http://dx.doi.org/10.1590/S1413-70542008000500016.

MARTINS, C.C. et al. Secagem e armazenamento de sementes de juçara. Revista Árvore, v. 33, n. 4, p. 635-642, 2009. http://dx.doi.org/10.1590/S010067622009000400006 . 
SILVA, A.C.D. et al. Morfologia e emergência de plântulas de $O$. bataua em diferentes substratos e luminosidades.

MARTINS, C.C.; MARTINELLI-SENEME, A.; NAKAGAWA, J. Estágio de colheita e substrato para o teste de germinação de sementes de ipê (Tabebuia chrysotricha (Mart. ex DC.) Standl.). Revista Árvore, v. 32, n. 1, p. 27-32, 2008. http://dx.doi.org/10.1590/ S0100-67622008000100004.

MENDONÇA, M.S. et al. Morfo-anatomia do fruto e semente de Oenocarpus minor Mart. (ARECACEAE). Revista Brasileira de Sementes, v. 30, n. 1, p. 90-95, 2008. http://dx.doi.org/10.1590/S010131222008000100012.

OLIVEIRA, A.B. et al. Emergência de plântulas de Copernicia hospita Martius em função do tamanho da semente, do substrato e do ambiente. Revista Brasileira de Sementes, v. 31, n. 01, p. 281-287, 2009. http://dx.doi.org/10.1590/S010131222009000100031.

PEREIRA, D.S.; SOUSA, J.E.S.; PEREIRA, M.S.; GONÇALVES, N.R.; BEZERRA, A.M.E. Influência da maturação dos frutos na emergência e crescimento inicial de Copernicia hospita Mart. - Arecaceae. Revista Ciência Agronômica, v. 45, n. 1, 2014.

PIVETTA, K.F.L. et al. Tamanho do diásporo, substrato e temperatura na germinação de sementes de Archontophoenix cunninghamii (Arecaceae). Revista de Biologia e Ciências da Terra, v. 8, n. 01, p. 126-134, 2008.

QUEIROZ, M.S.M.; BIANCO, R. Morfologia e desenvolvimento germinativo de Oenocarpus bacaba mart. (ARECACEAE) da Amazônia Ocidental. Revista Árvore, v. 33, n. 6, p. 1037-1042, 2009. http://dx.doi. org/10.1590/S0100-67622009000600006.
REIS, R.G.E. et al. Emergência e qualidade de mudas de Copernicia prunifera em função da embebição das sementes e sombreamento. Revista Caatinga, v. 24, n. 4, p. 43-49, 2011.

RODRIGUES, J.K.; MENDONÇA, M.S.; GENTIL, D.F.O. Efeito da temperatura, extração e embebição de sementes na germinação de Bactris maraja Mart. (Arecaceae). Revista Árvore, v. 38, n. 5, p. 857-865, 2014. http://dx.doi.org/10.1590/S010067622014000500010 .

SILVA, B.M.S.; CESARINO, F.; PANTOJA, T.F. Emergência de plântulas de Oenocarpus minor Mart. em diferentes profundidades de semeadura. Revista Brasileira de Agroecologia, v. 2, n. 1, p. 1329-1332, 2007.

SILVA, B.M.S. CESARINO, F.; PANTOJA, T.F. MÔRO, F.V. Germinação de sementes e emergência de plântulas de Oenocarpus minor Mart. (Arecaceae). Revista Brasileira de Fruticultura, v. 28, n. 2, p. 289-292, 2006b. http://dx.doi.org/10.1590/S010029452006000200030.

SILVA, V.L. et al. Morfologia e avaliação do crescimento inicial de plântulas de Bactris gasipaes Kunth. (Arecaceae) em diferentes substratos. Revista Brasileira de Fruticultura, v. 28, n. 3, p. 477-480, 2006a. http://dx.doi.org/10.1590/S010029452006000300030.

TAVEIRA, L.R. et al. Morfo-anatomia de plântulas de carnaúba (Copernicia prunifera (Mill.) H.E. Moore - Arecaceae). Revista de Ciências da Amazônia, v. 1, n. 1, p. 37-47, 2013. 